\title{
Laboratory Measurement of Heat Transfer and Thermal Structure Near an Air-Water Interface
}

\author{
ROBERT H. HILL \\ U. S. Naval Research Laboratory, Washington, D. C. 20390 \\ (Manuscript received 11 October 1971, in revised form 28 December 1971)
}

\begin{abstract}
Experiments have been conducted in a wind-water tunnel to establish a quantitative relationship between wind profile parameters, heat flux through the air-water interface, and the temperature difference across the water thermal boundary layer as observed with an infrared radiometer. Two distinct regimes of boundary layer characteristics were identified which are separated by a transition that coincides with the onset of surface waves. At low wind speeds the boundary layer can be characterized as laminar and a relatively large temperature difference is observed; a surface-active film enhances the temperature difference. At higher wind speeds, when the surface is roughened by waves, the boundary layer appears to become turbulent, resulting in a marked decrease in the temperature difference across the layer. Based on these results, estimates of sea surface, boundary-layer temperature differences agree with the few published field measurements. By using a simple laminar model of the water boundary layer, estimates are made of the variation in layer thickness with wind speed.
\end{abstract}

\section{Introduction}

Much interest has been shown in recent years in accurate determinations of sea surface temperature using remote infrared sensors. One of the problems involved in such measurements is uncertainty about the nature of the thermal boundary layers occurring at the air-sea interface. These boundary layers occur because turbulent mixing processes are suppressed near a boundary and the exchange coefficients for heat and water vapor approach molecular values at the interface, resulting in very steep temperature gradients within a few millimeters of the surface. Consequently, the surface radiation temperature generally differs signifcantly from the so-called "bucket" temperature obtained at a depth of several centimeters. The difference depends, of course, upon the total heat flux through the surface and, also, upon such factors as the surface wave field, subsurface flow, and the presence of surface active materials (slicks).

The small scale of these thermal boundary layers makes their study at sea from moving platforms quite difficult. Laboratory experiments provide a means of circunventing some of these difficulties but unrealistical modelling of conditions encountered at the sea surface (such as wind action, subsurface turbulence, and surface waves) often occurs. The purpose of the experiments discussed here was to obtain a quantitative relationship between heat flux through and thermal structure across an air-water interface under the conditions referred to above.

\section{Apparatus and procedure}

The experiments were conducted in a simple openwind tunnel, placed above a water tank such that the free water surface formed part of the tunnel floor. The overall length of the tunnel was approximately $3.6 \mathrm{~m}$ and the working cross section was $40.6 \mathrm{~cm}$ by $40.6 \mathrm{~cm}$. Turbulence levels within the tunnel were minimized by locating the fan at the downstream end and by providing a smoothly flared inlet and honeycomb at the upwind end. Midstream velocity $\left(\bar{U}_{S}\right)$ was continuously variable between 200 and $1100 \mathrm{~cm}$ $\mathrm{sec}^{-1}$. The tank was $43 \mathrm{~cm}$ deep and the free water surface $40.6 \mathrm{~cm}$ wide by $89.5 \mathrm{~cm}$ long. The system was adjusted so that the air flow encountered a smooth transition from the tunnel floor onto the water surface and the resultant waves were damped on a small "beach" at the downstream end. The walls and bottom of the tank were thermally insulated to insure that any heat transfer occurring there would be negligible compared to that through the water surface.

\section{a. Air velocily profiles}

Vertical profiles of air velocity were obtained with a hot-wire anemometer which employed a thin boundary layer probe, shown in Fig. 1, supported by a vertical traversing device. Air velocity measurements were made at distances of $4.4,42.5$ and $80.6 \mathrm{~cm}$ from the leading edge of the water surface, and at eleven heights above mean water level varying from 0.5 to $6.0 \mathrm{~cm}$ at intervals that increased upward approximately logarithmically. 
Above $6 \mathrm{~cm}$, the midstream velocity was essentially constant up to very near the tunnel roof. During these measurements water was continually added to the tank to maintain the surface level against evaporation losses.

Although means were not available for making quantitative surface wave measurements, two other types of observations related to wind stress on the water surface were made. Because unfiltered tap water was used in the tank there always existed a thin film of material on the surface which would be compacted in the downwind end of the tank under the action of wind stress. At relatively low wind speeds the upwind edge of the film was visible and its position as a function of midstream air velocity was recorded. In addition, at higher wind speeds the water surface would become ruffled by waves; the fetch at which this occurred was clearly defined and also was recorded as a function of midstream velocity.

\section{b. Heat flux}

Two types of temperature measurements were made to properly control the experiment and to provide a basic parameter of interfacial heat transfer. One type was obtained using thermistor sensors mounted in two kinds of probes as shown in Fig. 1. These sensors provided a continuous record of absolute temperature at several points in the air stream and water tank and, in addition, were connected differentially to provide the air-water temperature difference and to check against significant gradients in the bulk water. A second type of measurement was made which provided water surface temperature at several points relative to that of the bulk water. This was accomplished with thermocouple probes in the form of vertical loops supported beneath

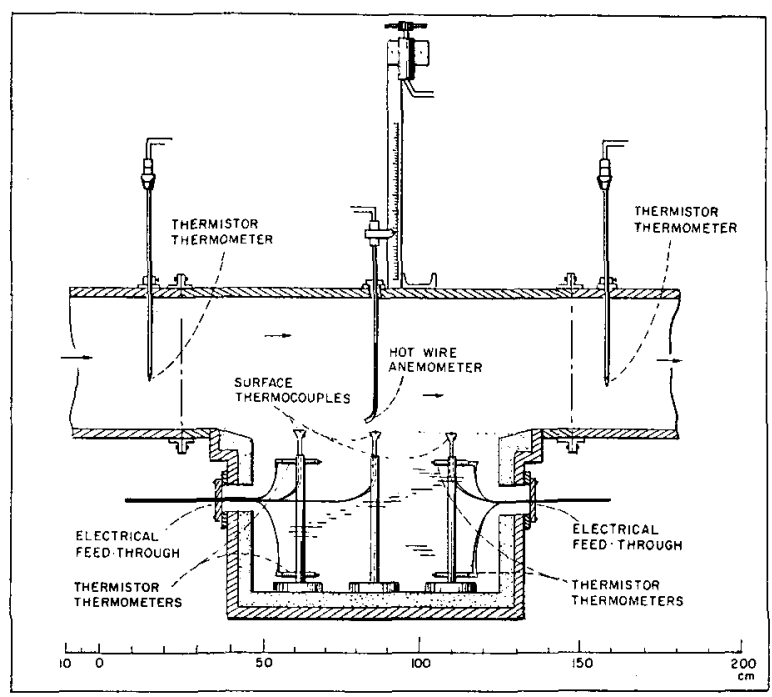

Frg. 1. Arrangement of temperature and air speed sensors. The hot-wire anemometer was used at various distances from the upwind edge of the water.

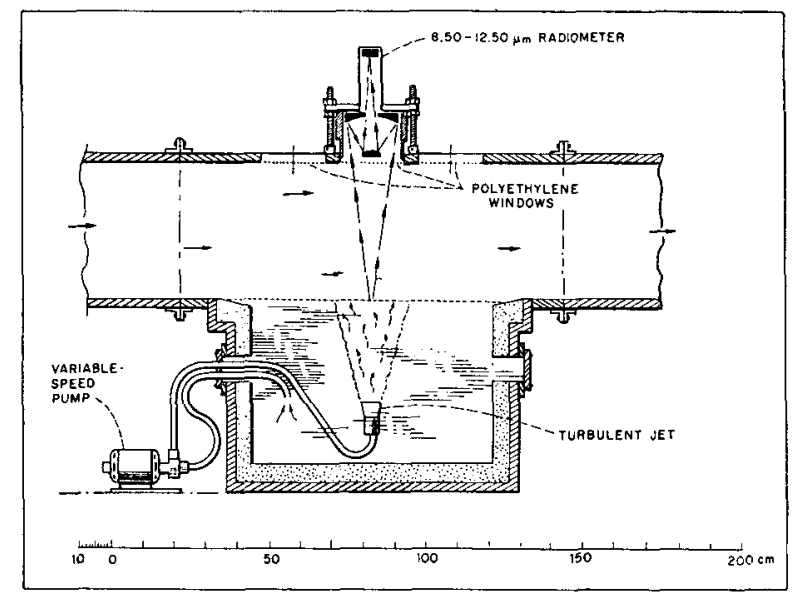

FIG. 2. Configuration of the infrared radiometer and submerged turbulent jet used to determine the temperature difference across the water thermal boundary layer.

the water surface, as shown in Fig. 1, such that one junction of the thermocouple pair was in the surface (forming a slight meniscus in an otherwise undisturbed surface) and the second, or reference, junction was at a depth of $4 \mathrm{~cm}$. These measurements, used in estimating mean surface temperatures employed in heat flux calculations, generally agreed closely with the radiometric measurements described below but were affected by changes in water level due to evaporation and by the larger amplitude surface waves.

Mean values of total heat flux through the interface were obtained from a calorimetric calculation based on the change in bulk water temperature with time, the measured heat capacity of the tank contents, and the water surface area. Mean latent heat flux was derived from total evaporation measurements using an interferometric optical system to determine, to within about $0.005 \mathrm{~mm}$, the change in water level in a small open reservoir connected to the experimental tank. Since evaporation is directly related to the difference in water vapor pressure between the surface and the otherlying air, an aspirated dew point hygrometer was used upwind of the water tank to determine the water vapor content of the air. A simple horizontal flux-plate net radiometer, $9.0 \mathrm{~cm}$ by $11.5 \mathrm{~cm}$, was used to determine radiant heat flux.

\section{c. Thermal structure}

Of primary interest in these experiments was the measurement with an infrared radiometer of water surface temperature relative to the temperature at a depth of a few centimeters as a function of heat flux and wind stress at the interface. The radiometer, shown in Fig. 2, viewed the water surface at normal incidence through thin polyethylene windows built into the tunnel roof. The optical bandpass of the instrument was 8.5 to $12.5 \mu \mathrm{m}$; the absorption coefficient of water at these wavelengths is such that the apparent radiation 


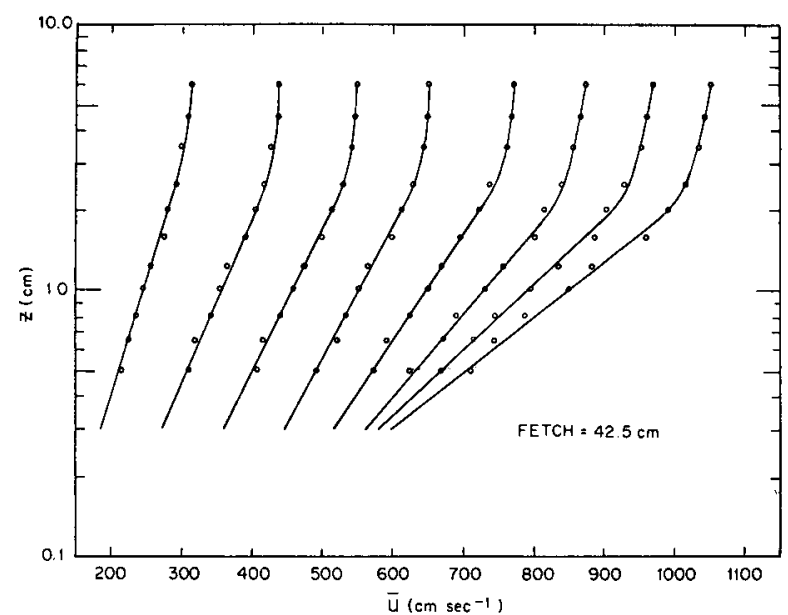

FIg. 3. Typical wind velocity profiles.

temperature of a water surface is equal to the actual temperature at a depth of approximately $0.01 \mathrm{~mm}$. The field of view was about $1 \mathrm{~cm}^{2}$ in area and temperature changes of the order of $0.01 \mathrm{C}$ could be resolved.

A submerged turbulent jet was placed in the water tank directly beneath the radiometer field of view as shown in Fig. 2. The temperature difference across the water thermal boundary layer was determined by first viewing the undisturbed surface and then removing the boundary layer with the submerged jet. These measurements were made at distances of $17.9,44.7$ and $74.3 \mathrm{~cm}$ from the upwind edge of the water surface and over a wide range of heat flux values.

\section{Discussion of results}

In the following discussion spatial dimensions are referenced to a coordinate system with the origin at the upwind edge of the water, the positive $X$ axis (fetch) extending downwind at mean water level, and the $Z$ axis being positive upward. Because lateral symmetry was carefully maintained in the apparatus and because wave crests and the edges of compressed surface films were observed to be normal to the flow direction except very near the walls, the flow was assumed to be twodimensional to a good approximation.

\section{a. Air velocity profiles}

Wind action on the sea surface is the controlling factor in virtually all exchange processes occurring at the interface. Consequently, to establish a basis for comparisons between laboratory and oceanic conditions, vertical profiles of mean wind speed were measured as described earlier; a typical set of profiles is shown in Fig. 3. On this semi-logarithmic plot the data approximate a straight line up to slightly more than $2 \mathrm{~cm}$; this is indicative of a developing turbulent boundary layer. Moreover, the anemometer output showed the air stream to be fully turbulent at all levels. A least- squares fit was obtained for the data points within the boundary layer using the well-known expression

$$
\bar{U}=\left(U_{*} / k\right) \ln \left(Z / Z_{0}\right), Z \gg z_{0},
$$

where $\bar{U}$ is the mean wind speed at height $Z$, and $k$ von Kármán's constant (taken to be 0.4). The profile parameters $U_{*}$ and $Z_{0}$ are termed, respectively, the friction velocity (obtained from the slope of the profile) and the roughness length (obtained from the $Z$ intercept). For a given profile, $U_{*}$ is constant (for neutral stability conditions) and is considered to be a more meaningful characterization of the profile than, say, the mean wind speed at an arbitrarily specified height. The roughness length exhibits a complex dependence on the boundary over which the flow occurs.

The problem of scaling between laboratory and field observations of wind profiles, surface waves, and other air-sea interactions has been investigated in windwave tanks. The form of (1) is generally accepted as a valid representation of wind profiles over water surfaces in the laboratory and above the sea surface under conditions of near-neutral stability, but with some uncertainty remaining concerning the nature of the roughness length $Z_{0}$. For smooth flow conditions where the surface characteristics are dominated by capillary waves, Wu (1968) suggested that

$$
Z_{0}=b \frac{\sigma}{\rho U_{*}^{2}}
$$

where $\sigma$ is the surface tension, $\rho$ the water density, and $b$ a proportionality constant roughly equal to 0.18 [with the wind profile expressed in the form of (1) ]. Charnock (1955) suggested, based on a dimensional argument, that

$$
Z_{0}=a U_{*}^{2} / g
$$

where $g$ is the gravitational acceleration and $a$ is a constant of proportionality. This expression has been found to hold for rough flow conditions in laboratory tanks if sufficient fetch (a few meters) is allowed for equilibrium to be established between the wind profile and surface waves and with $a=0.011$ (Hidy and Plate, 1966). It has also been found to be consistent with field observations ( $\mathrm{Wu}, 1968)$.

The variation of friction velocity, according to (1), with midstream velocity, measured at $Z=6 \mathrm{~cm}$, is shown in Figs. 4a-c. As discussed below, the flow conditions changed from aerodynamically smooth to rough within this wind speed range. At both the upwind and downwind positions $U_{*}$ is seen to increase quite uniformly with $\bar{U}_{S}$. However, at the intermediate fetch the rate of increase is discontinuous at one point; the discontinuity corresponds to the transition from a smooth to a rippled surface. It is thought that the horizontal drift velocity of the water surface, which would be much larger near the tank center than near 
the ends, would account for the reduced values of $U_{*}$ at the intermediate fetch. The transition from aerodynamically smooth to rough flow is demonstrated in

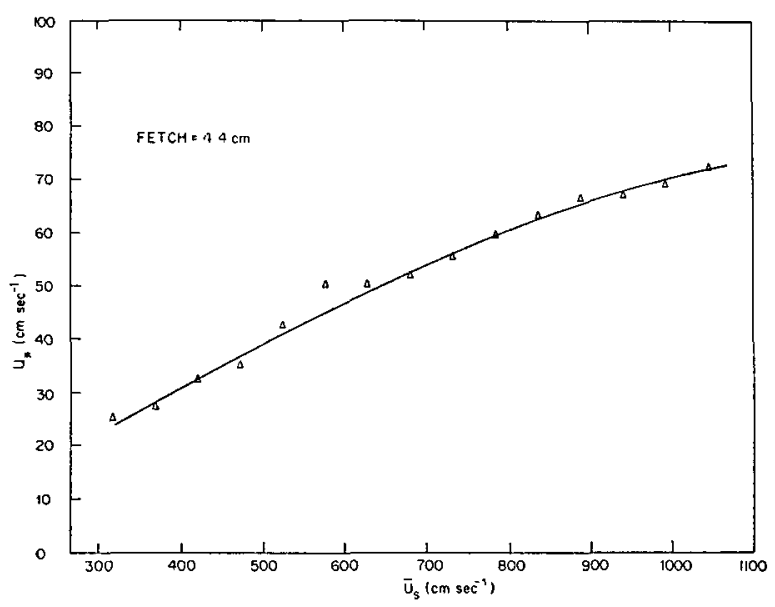

a.

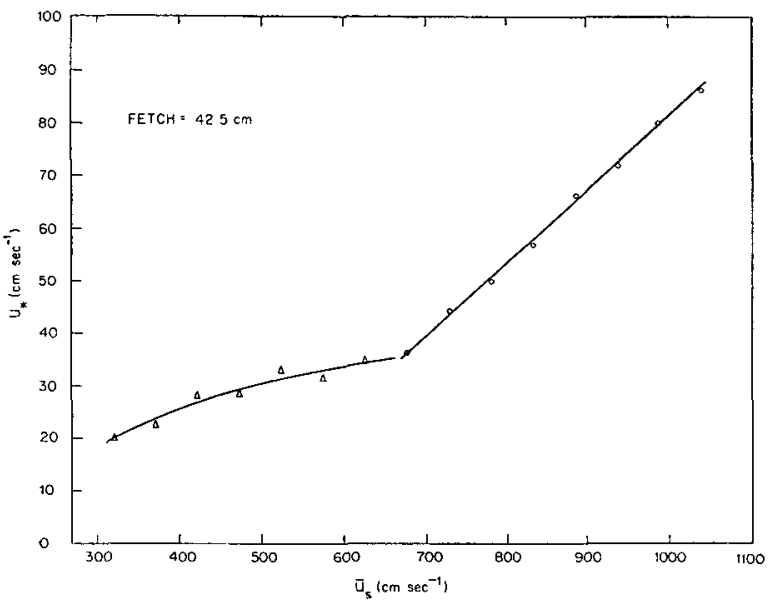

b.

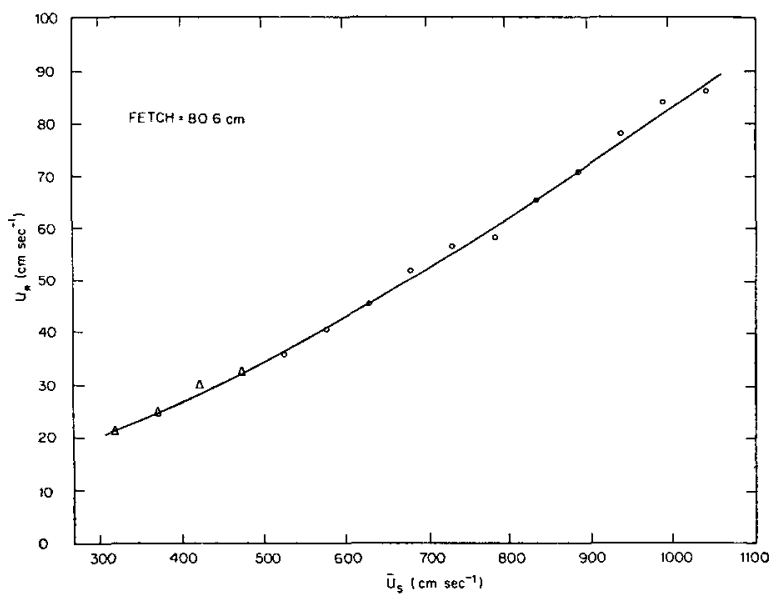

c.

FIg. 4. Variation of friction velocity with midstream air velocity at three values of fetch. Flow conditions changed from smooth (triangles) to rough (circles).

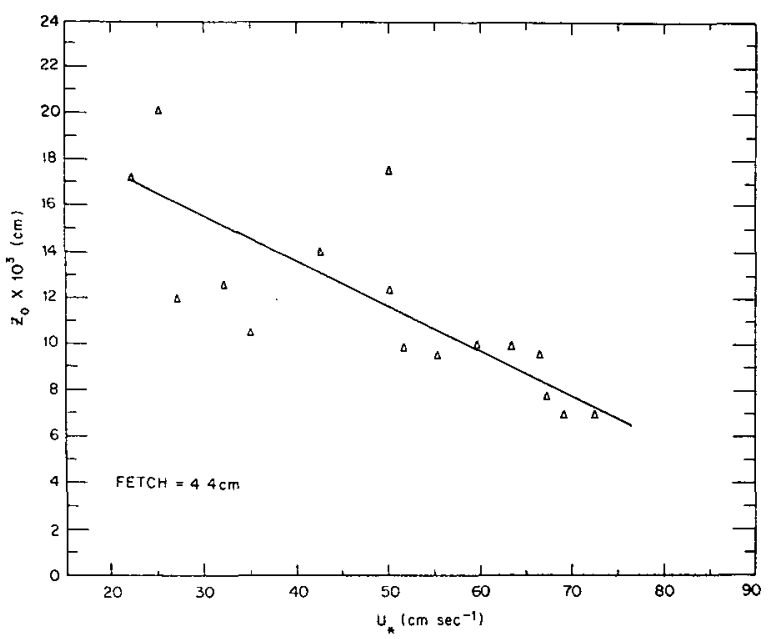

a.

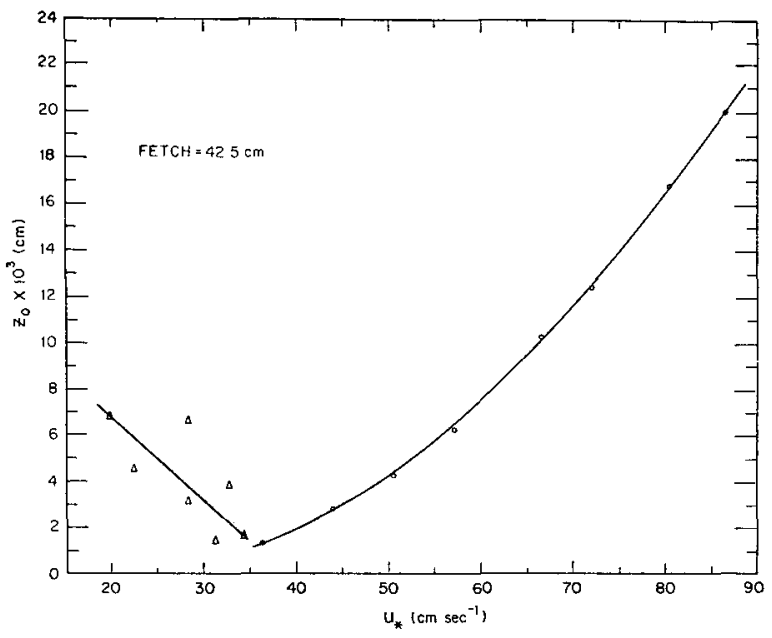

b.

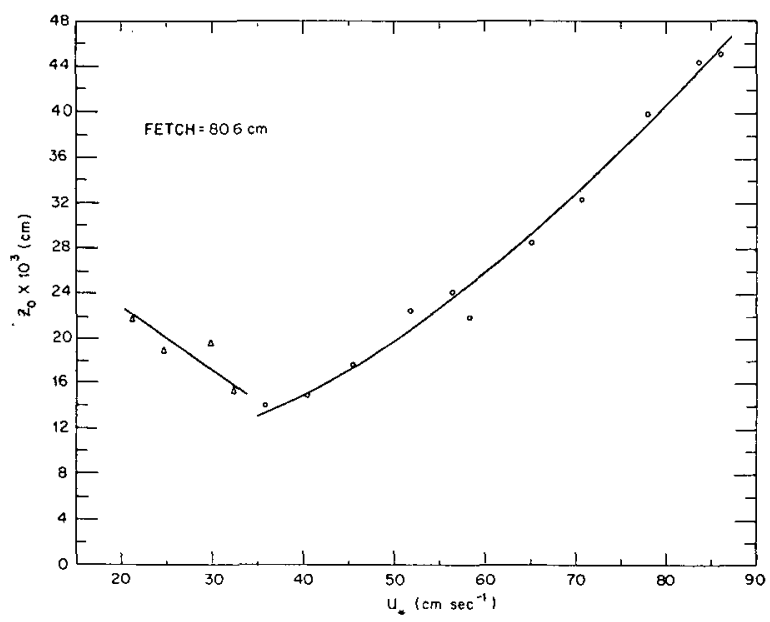

c.

FIG. 5. Same as Fig. 4 except for variation of roughness length with friction velocity.

Figs. 5a-c which show the variation of roughness length with friction velocity. The data obtained at a 


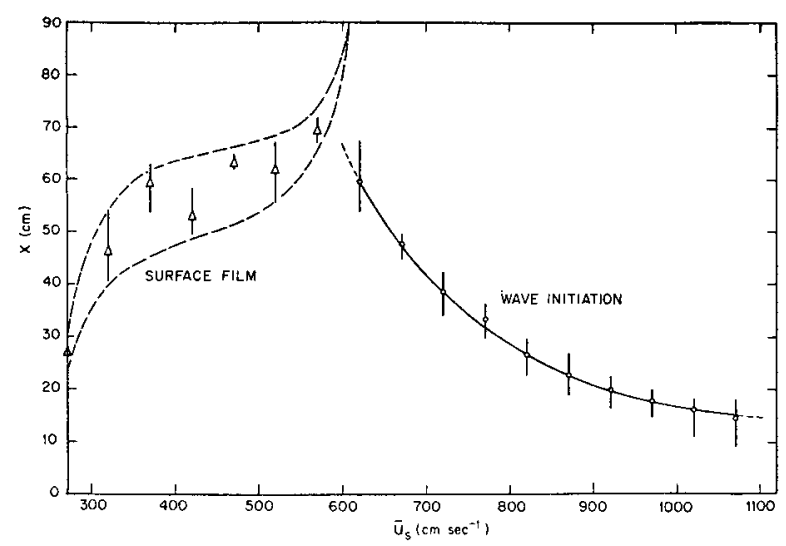

FIG. 6. Fetch to edge of surface film showing compaction of film, and critical fetch necessary to generate visible waves as a function of midstream air velocity.

fetch of only $4.4 \mathrm{~cm}$ show a continuous decrease in $Z_{0}$ which is characteristic of smooth flow and was probably determined more by the upwind tunnel floor than by the water surface. At the intermediate and downwind positions the change in the nature of the flow is evident and coincided with the onset of surface waves at each particular value of fetch.

There appears to be general agreement between these results and those cited above concerning the manner in which the roughness length varies with friction velocity and the value of friction velocity at which the transition occurs. How ever, although the values of Charnock's proportionality constant computed from these data are of the correct order, the roughness length varies with fetch (note that the ordinate scale of Fig. $5 \mathrm{c}$ is compressed), indicating that equilibrium conditions were not achieved due to the short wave tank.

As mentioned earlier, compression of the surface film and the onset of capillary ripples and short gravity waves were visually observed and recorded as a function of $\bar{U}_{S}$. Shown in Fig. 6 is the fetch to the edge of the film and also the fetch necessary to raise waves. Each data point is the average of many observations over a range indicated by the vertical bar through each point. The amount of material in the surface film was not known and undoubtedly varied in each case. Apparently the material was merely being consolidated up to $\bar{U}_{S} \approx 350 \mathrm{~cm} \mathrm{sec}^{-1}$ and thereafter the film was being compressed under the action of increasing surface stress. At $\bar{U}_{S} \approx 550 \mathrm{~cm} \mathrm{sec}{ }^{-1}$ the film would collapse and be deposited on the downwind beach. Wave initiation at the lowest speed at which waves appeared was obscured somewhat by the small remaining surface film but was well defined at higher speeds. Wavelengths of the initial ripples were of the order of $1 \mathrm{~cm}$ but grew to over $10 \mathrm{~cm}$ at maximum fetch and wind speed. No means were available for measuring wave amplitudes, but, again at maximum fetch and wind speed, the amplitude never exceeded $8 \mathrm{~mm}$ judging by the lowest level at which air velocity measurements could be made.

\section{b. Heat fux}

Total, latent and net radiant heat flux through the air-water interface were measured directly, as discussed earlier, using, respectively, a calorimetric technique, evaporation determinations, and a net radiometer. Sensible heat flux was calculated by two methods: as the residual part of the total heat flux, and from the latent flux alone using the Bowen ratio. The Bowen ratio is simply the ratio of sensible to latent heat flux [see Roll (1965) for a relevant discussion] and, for typical environmental conditions, is expressed as

$$
B=H_{S} / H_{E}=0.66\left(T_{0}-T_{a}\right) /\left(e_{0}-e_{a}\right)
$$

where $T_{0}$ and $e_{0}$ are, respectively, the temperature $\left({ }^{\circ} \mathrm{C}\right)$ and saturation water vapor pressure $(\mathrm{mb})$ at the sea surface, and $T_{a}$ and $e_{a}$ are the temperature and water vapor pressure at some height above the surface. This relationship, which is independent of wind speed, has been found to be valid for heat transfer over natural bodies of water, and was tested and found to hold for the laboratory case discussed here as well.

The variation of latent heat flux with friction velocity is shown in Fig. 7. Since each value applies to the entire water surface, the mean value of the friction velocities obtained at the three fetches was used. Vapor pressure differences were varied over as wide a range as ambient humidity variations and limitations on water temperature would allow; each measurement was normalized by dividing by the corresponding vapor pressure difference. The data were fitted with the linear expression

$$
\left(H_{e} \times 10^{3}\right) /\left(e_{0}-e_{a}\right)=0.02 \bar{U}_{*},
$$

where $\bar{U}_{*}$ is the mean friction velocity, $e_{0}$ the saturation vapor pressure corresponding to the mean surface temperature, and $e_{a}$ the vapor pressure corresponding to dew point measurements obtained near the tunnel inlet. There appeared to be no significant modification of the evaporation process by either the surface film or waves. It has been found that, due to inevitable impurities or insufficient compaction, naturally occurring surface

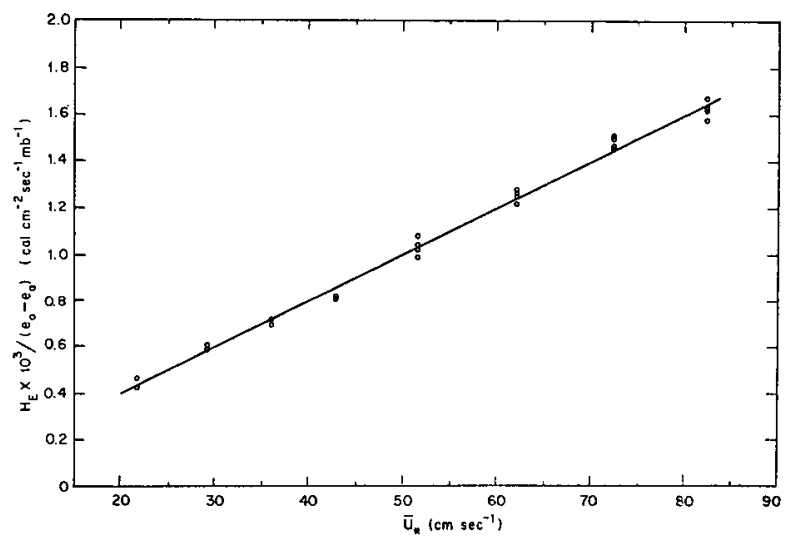

FIG. 7. Variation of normalized latent heat flux with mean friction velocity. 
films will not appreciably retard evaporation (Barger and Garrett, 1968). However, Easterbrook (1969) has found, for certain combinations of wind speed and surface waves, that evaporation is reduced in comparison to the same wind speed conditions but with no waves. Kanwisher (1963) made a similar observation relative to gas diffusion above the interface. The effect is attributed to flow separation behind gravity wave crests which alters exchange coefficients in the air near the water surface. Apparently the waves in the laboratory tank were never sufficiently developed for this process to occur.

Sensible heat flux as a function of friction velocity is shown in Fig. 8. The curve is calculated from the curve of Fig. 7 using the Bowen ratio; the data points were obtained by subtracting latent and net radiant heat flux from the total. The large scatter in the data resulted from the fact that, for this particular set of measurements, humidity was low and latent heat flux greatly predominated over sensible heat flux (a typical value for the Bowen ratio was -0.067$)$; consequently, the error in the difference between total and latent heat flux was comparable to the sensible heat flux itself. (The original check on the validity of the Bowen ratio for the laboratory case was conducted earlier under much more favorable circumstances, i.e., humidity was much higher so that latent heat flux was proportionately smaller; these data are not plotted in Figs. 7 and 8 because wind profile parameters were not determined simultaneously.)

The net radiant heat flux $H_{R}$ measured near the water surface was found to be represented by

$$
\left(H_{R} \times 10^{3}\right) /\left(T_{0}-T_{a}\right)=0.14,
$$

where $T_{0}$ is water surface temperature, and the tunnel interior is assumed to be at air stream temperature $T_{a}$. The radiation characteristics of the wind-water tunnel were approximately equivalent to conditions at sea when the sky is heavily overcast and the atmosphere has a high water vapor content. For different conditions (e.g., clear sky and light wind) net radiation can be the dominant mode of heat flux.

\section{c. Thermal siructure}

The temperature difference across the water thermal boundary layer was determined with an IR radiometer by first viewing the undisturbed water surface and then removing the boundary layer with a submerged turbulent jet. Inherent in this method is the assumption that the vertical extent of the thermal boundary layer is always large when undisturbed compared to the equivalent depth $(0.01 \mathrm{~mm})$ for which the radiation temperature is obtained, and is always very small above the turbulent jet. Although the heat flux was varied over quite a wide range during the observations discussed here, for each case the total heat flux was from water to air producing a relatively cool surface layer.

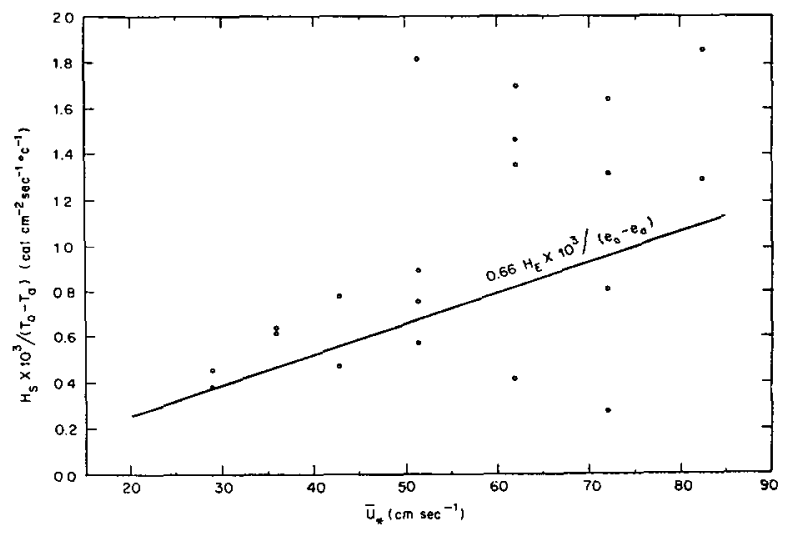

Fig. 8. Variation of normalized sensible heat flux with mean friction velocity. The curve is calculated from the latent heat flux using the Bowen ratio.

A prominent feature of these data was a large decrease in the boundary-layer temperature difference which occurred with the onset of surface waves and the change from aerodynamically smooth to rough flow as evidenced by the wind profile. In addition, the presence of a surface film at the lowest wind speeds would significantly increase the temperature difference. In spite of the negative density stability of these cold layers, temperature differences could be maintained which were of the order of $1.0 \mathrm{C}$ through the smooth regime, and even larger across the surface film. A limiting temperature difference, assuming one exists, was not determined.

To relate the boundary-layer temperature differences to total heat flux and the wind profile, an expression in the form of a heat transfer equation was written first as

$$
H_{W}=\gamma \rho_{w} C_{p}\left(T_{w}-T_{0}\right) V_{*}
$$

where $H_{W}$ is the total heat flux through water boundary layer, $\gamma$ the heat transfer coefficient, $\rho_{w}$ the water density, $C_{p}$ the isobaric specific heat of water, $T_{w}$ the bulk water temperature, $T_{0}$ the water surface temperature, and $V_{*}$ an effective friction velocity associated with the water boundary layer. Thus, by definition, we have

$$
\begin{gathered}
H_{W}=H_{T}, \\
T_{w}-T_{0}=-(\Delta T)_{R},
\end{gathered}
$$

where $H_{T}$ is the total heat flux above the water surface and $(\Delta T)_{R}$ denotes the temperature difference across the water thermal boundary layer as determined with the infrared radiometer. The quantity $V_{*}$ was scaled from the wind profile by

$$
V_{*}=\left(\rho_{a} / \rho_{w}\right)^{\frac{1}{2}} U_{*},
$$

where $\rho_{a}$ and $U_{*}$ are the air density and friction velocity, respectively. Eq. (8c) implies a turbulent, quasisteady, water boundary layer, not necessarily always the case, and ignores any stress supported by waves; 
these discrepancies will be absorbed in the coefficient $\gamma$. Eq. (7) now becomes

$$
H_{T}=-\gamma C_{p}\left(\rho_{a} \rho_{w}\right)^{\frac{1}{2}}(\Delta T)_{R} U_{*},
$$

and the properties of the boundary layer can be examined in terms of the coefficient $\gamma$. During the radiometric measurements all of the parameters necessary for the determination of total heat flux were monitored. By combining (4), (5) and (6), $H_{T}$ in Eq. (9) could be calculated.

In Fig. 9 the reciprocal of the water boundary layer heat transfer coefficient, calculated from data obtained at a fetch of $44.7 \mathrm{~cm}$ with different values of heat flux, is plotted against the local friction velocity. (The reciprocal of $\gamma$, a nondimensionalized form of the temperature differences, gives a better representation of its variations.) The transition between the smooth and rough regimes occurring at $\bar{U}_{*} \approx 35 \mathrm{~cm} \mathrm{sec}^{-1}$, and the effect of the surface film at the lowest wind speed are quite evident. The coefficient is seen to have characteristic values for each of these states which are independent of heat flux. Shown in Fig. 10 are similar data obtained near the upwind $(X=17.9 \mathrm{~cm})$ and downwind $(X=74.3 \mathrm{~cm})$ ends of the water surface. The upwind data, not influenced by the surface film, show the variation from smooth to rough surface conditions, whereas the downwind data show the influence of the film and, after it collapsed, the rough surface condition. Although the transitions from one condition to another are not as well defined, the initial and final values for each compare well with those obtained near the tank center. At least for the rough condition the heat transfer coefficient appears to steadily increase with wind speed.

The suppression of turbulent eddies subjacent to an air-water interface by surface active films has been treated theoretically by Davies (1966), and the effect of surface films on the water thermal boundary layer has been examined experimentally by Jarvis (1962). Although materials of a particular chemical class, if sufficiently pure and highly compacted, can affect the surface thermal structure directly by suppressing evaporation, virtually any surface active material, even in small quantities, will suppress near-surface convective motion (by altering surface tension and viscosity) with a consequent increase in the temperature difference across the water thermal boundary layer. Since no effect on evaporation rate was noted in the present experiment, the latter process affords an explanation for the large temperature differences observed with a surface film present. This conclusion was reinforced by the observation that dust particles on the water surface moved rapidly downstream across a clean surface but exhibited slow, random-like motion when in a surface film.

A transition from laminar to turbulent flow conditions in the water boundary layer appears to be responsible for the difference in thermal structure between the

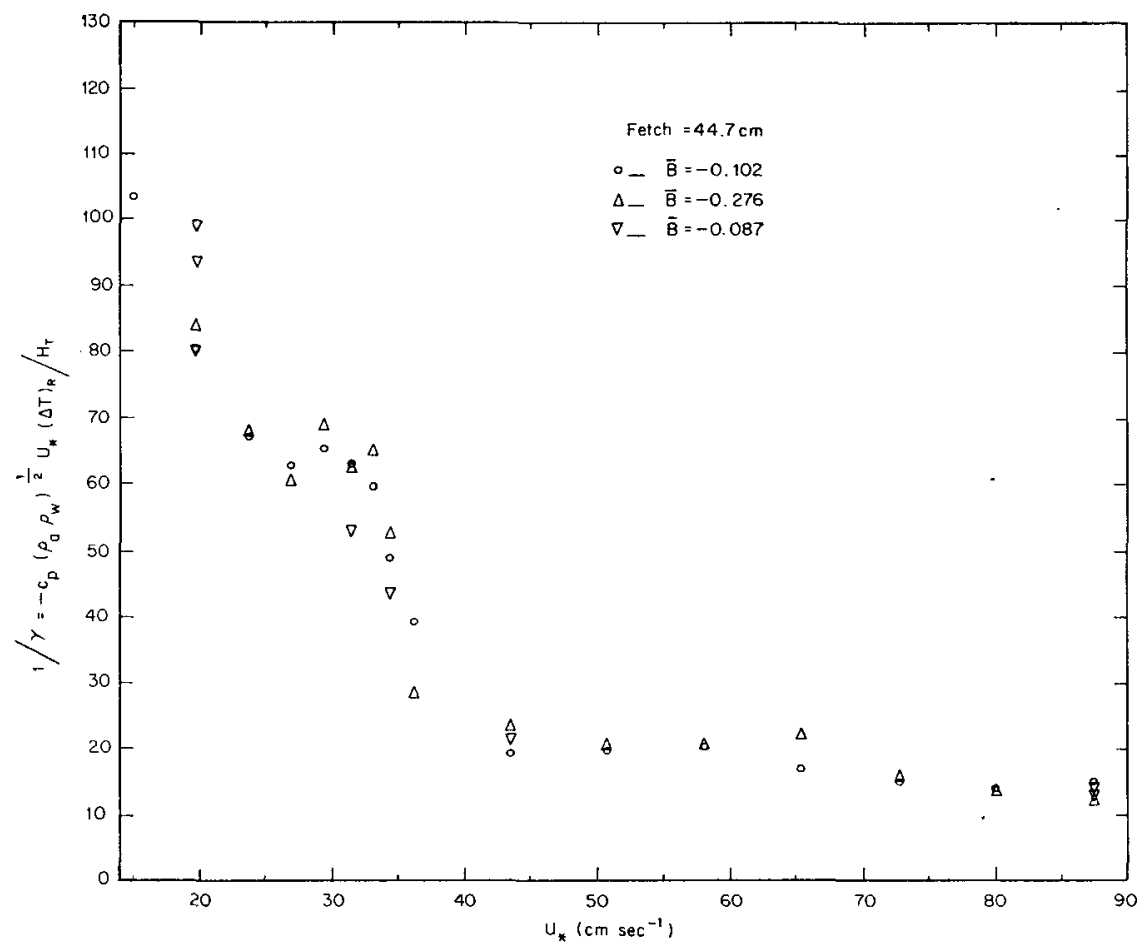

Fig. 9. Variation of the reciprocal of water boundary layer heat transfer coefficient $(\gamma)$ with local air-friction velocity for different Bowen ratios $(\bar{B})$, showing surface film effects and the transition from smooth to rough conditions. 


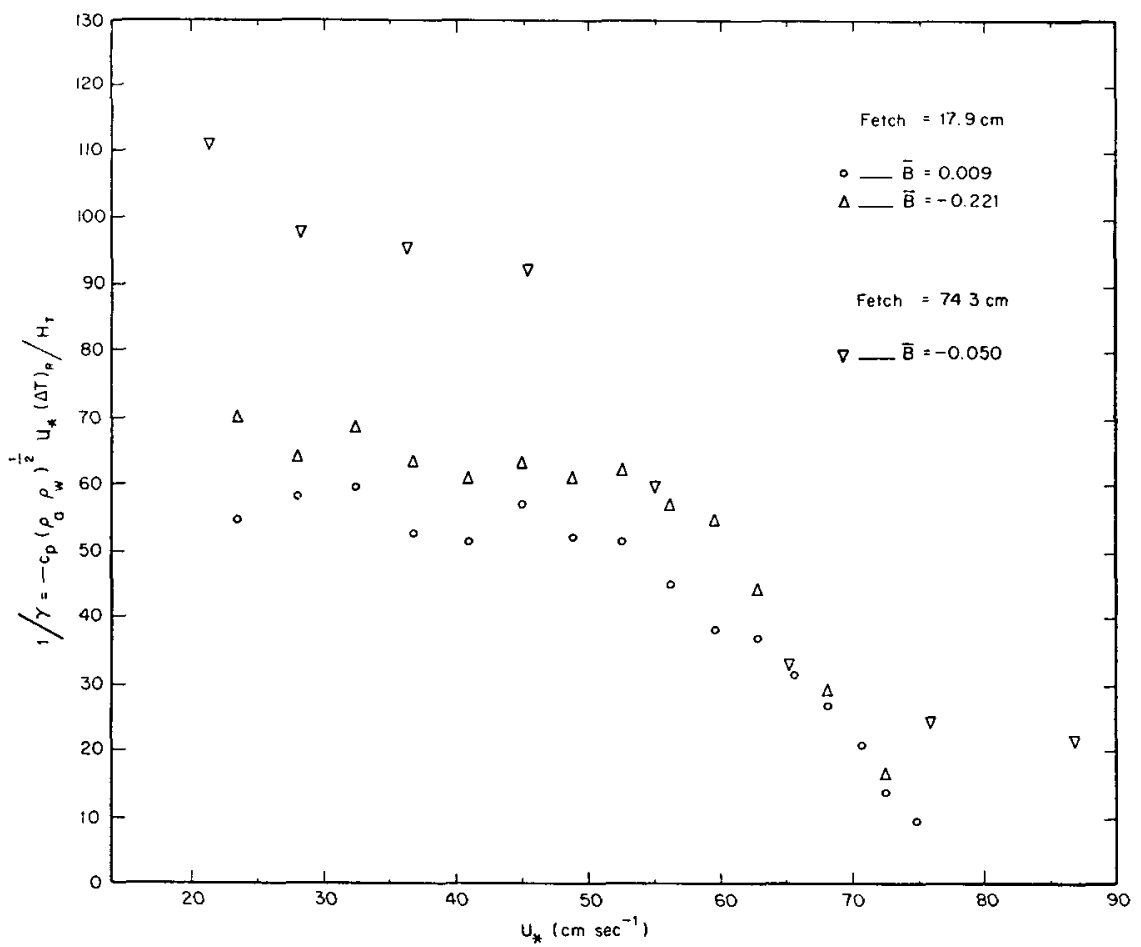

Fig. 10. Variation in the reciprocal of water boundary layer heat transfer coefficient $(\gamma)$ with local air-friction velocity for different Bowen ratios $(\bar{B})$. Upwind data show the variation from smooth to rough conditions with increasing wind speed, and downwind data the surface film effect.

smooth and rough regimes referred to previously. Several investigators have supplied ample evidence that such a transition occurs. Plate et al. (1969), using hydrogen bubble tracers, found that the water boundary layer became turbulent as visible waves appeared. McAlister and McLeish (1969) have observed the change in thermal structure, and Kanwisher (1963) has observed an increase in the rate of gas diffusion across the water boundary layer as the surface is roughened by ripples and short waves.

A frequently employed model of the water thermal boundary layer is one in which the surface layer possesses a linear temperature gradient to a depth $\delta$ below which the fluid is always well mixed and has no temperature gradient. Of basic importance then are estimates of the layer thickness $\delta$. To obtain such estimates from these data, an expression for conduction through the layer was written as

$$
H_{w}=-k_{w} \frac{(\Delta T)_{R}}{\delta}
$$

where $k_{w}$ is the thermal conductivity of water; combining (10) with (8a) and (9), we obtain

$$
\delta=\frac{k_{w}}{C_{p}\left(\rho_{a} \rho_{w}\right)^{\frac{1}{2}}}\left(\frac{1}{\gamma U_{*}}\right)
$$

The variation of $\delta$ with friction velocity is shown in Fig. 11, based on data obtained at a fetch of $44.7 \mathrm{~cm}$. Of particular interest are the small values of $\delta$ in the rough regime $\left(U_{*}>35 \mathrm{~cm} \mathrm{sec}^{-1}\right)$ which range down to $0.07 \mathrm{~mm}$ at the highest wind speeds. Although this simple boundary model probably has limited applica-

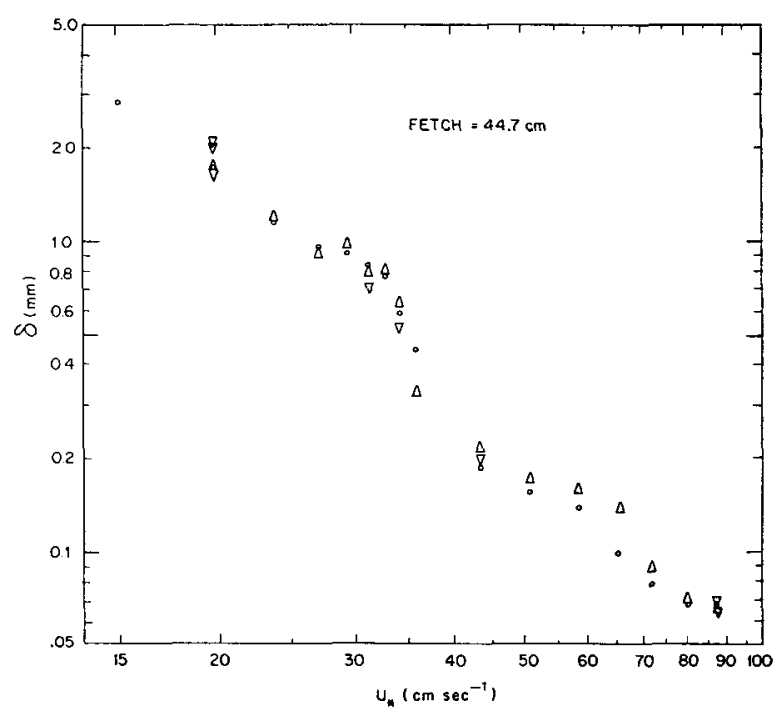

FIG. 11. Variation in calculated water boundary layer thickness with local air-friction velocity. 
bility at higher wind speeds, these low values of $\delta$ indicate the critical connection between the nature of the water boundary layer and the effective depth to which radiometric temperature measurements refer. McAlister and McLeish (1969) have discussed this problem with regard to radiometric observations for effective depths of $0.025,0.075$ and $0.500 \mathrm{~mm}$.

\section{Conclusions}

Laboratory measurements have provided a quantitative relationship between heat flux, wind profile parameters, and thermal structure near an air-water interface. Also, two regimes of water boundary layer heat transfer characteristics have been identified which are separated by a transition which coincides with the onset of surface waves. Due to the short fetch and consequent nonequilibrium conditions involved in these experiments between the wind profile and surface waves, it is difficult to predict with confidence at what wind speed such a transition might occur on the sea surface. Based on these results, one would expect it to occur with the first appearance of capillary and short gravity waves. However, Boudreau (1965) has presented data obtained in the Gulf of Mexico which indicate a marked reduction in boundary layer temperature difference occurring at a wind speed of $\sim 12 \mathrm{kt}$. It is not known whether this was due to the transition discussed here or to increased subsurface turbulence associated with breaking waves which commence at about this wind speed.

Although there is uncertainty as to where the boundary layer transition might occur, the experimentally determined values of the heat transfer coefficient were employed in (9) to estimate boundary layer temperature differences that occur at the sea surface. The method given by James (1966) was used to calculate total heat flux for a few sets of typical environmental conditions. Eqs. (1), (2) and (3) were used to relate friction velocity to a mean wind speed at an anemometer height of $10 \mathrm{~m}$. At the lower wind speeds (small value of $\gamma$ ) the temperature differences ranged up to about $1.0 \mathrm{C}$ without a surface film, and up to almost $2.0 \mathrm{C}$ if the effect of a film was included. At higher wind speeds (large value of $\gamma$ ) the temperature differences were of the order of $0.1 \mathrm{C}$ or less. A few field measurements of water thermal boundary layer characteristics, such as those summarized by Roll (1965), have been made usually under relatively low wind speed conditions. The values calculated above for the low wind speed case appear to agree well with these. Although reliance upon such numerical values must await verification by further field measurements, it is interesting to note, in spite of generally increasing heat flux through the interface, that the boundary layer temperature difference decreases with an increase in wind speed.

Acknozoledgments. The invaluable assistance of $\mathrm{Mr}$. Charles W. Martz in setting up and conducting the experiment is acknowledged.

\section{REFERENCES}

Barger, W. R., and W. D. Garrett, 1968: Modifications of the air/sea interface by artificial sea slicks. Rept. 6762, Naval Research Lab., Washington, D. C.

Boudreau, R. D., 1965: Skin temperature of the sea as determined by radiometer. Tech. Rept., Dept. of Oceanography, Texas A\&M University.

Charnock, H., 1955: Wind stress on a water surface. Quart. J. Roy. Meteor. Soc., 81, 639-640.

Davies, J. T., 1966: The effects of surface films in damping eddies at a free surface of a turbulent liquid. Proc. Roy. Soc. London, A290, 515-526.

Easterbrook, C. C., 1969: A study of the effects of waves on evaporation from free water surfaces. Res. Rept. 18, Bureau of Reclamation (Government Printing Office).

Hidy, G. M., and E. J. Plate, 1966: Wind action on water standing in a laboratory channel. J. Fluid Mech., 26, 651-688.

James, R. W., 1966: Ocean thermal structure forecasting. ASWEPS Manuel Series, Vol. 5, U. S. Naval Oceanographic Office (Government Printing Office).

Jarvis, N. L., 1962: The effect of monomolecular films on surface temperature and convective motion at the water/air interface. J. Colloid Sci., 17, 512-522.

Kanwisher, J., 1963: On the exchange of gases between the atmosphere and the sea. Deep-Sea Res., 10, 195-207.

McAlister, E. D., and W. McLeish, 1969: Heat transfer in the top millimeter of the ocean. J. Geophys. Res., 74, 3408-3414.

Plate, E. J., P. C. Chang and G. M. Hidy, 1969: Experiments on the generation of small water waves by wind. J. Fluid Mech., $35,625-656$.

Roll, H. U., 1965: Physics of the Marine Atmosphere. New York, Academic Press, $426 \mathrm{pp}$.

$\mathrm{Wu}, \mathrm{J} ., 1968$ : Laboratory studies of wind-wave interactions. $J$. Fluid Mech., 34, 91-111. 